\title{
CIPROFLOXACIN IN NEONATOLOGY
}

F. Bennaoui ${ }^{1,2}$, N . El Idrissi Slitine ${ }^{1,2}$, F.M.R Maoulainine ${ }^{1,2}$

1. Neonatal Intensive Care Department, Mohammed VI University Hospital

2. Research Team for Childhood, Health and Development, Marrakech School of Medicine, Cadi Ayyad University, Marrakech

\section{Introduction:}

The use of quinolone in children is limited by the potential risk of growth cartilage toxicity and also by the risk of emergence of resistant strains.

But in specific cases, Ciprofloxacin has often been used in off-label pediatrics.

In neonatology its use, although rare, is allowed in case of life-threatening infection.

\section{Materials \& Methods :}

-Retrospective study, conducted at the neonatal resuscitation department, Mohamed VI University Hospital of Marrakech.

-Duration: 2 years and 8 months from January 2015 to September 2017.

-The population of our study: all newborns treated with quinolone during their stay in the service.

\section{Results:}

-We collected thirteen cases, which were included during the study period, including 9 girls and 4 boys

-The average age at admission was 3.7 days.

-Ciprofloxacin was used in 2nd line after failure of conventional antibiotic therapy in all our patients.

-The dose administered was $20 \mathrm{mg} / \mathrm{kg}$ twice daily in 13 infants. The average duration of intra-hospital treatment was 13 days. The treatment protocol with ciprofloxacin in our department recommends a duration of ten to 21 days intravenously relayed orally for six to eight weeks if ventriculitis.

-The indications are illustrated in Chart II, for nosocomial infection the isolated organisms were klebsiella pneumonia in 3 cases, Enterbacter cloacae in 2 cases, the association klebsiella pneumonia with Enterbacter cloacae in one case and a case of Acinetobacter Baumani.

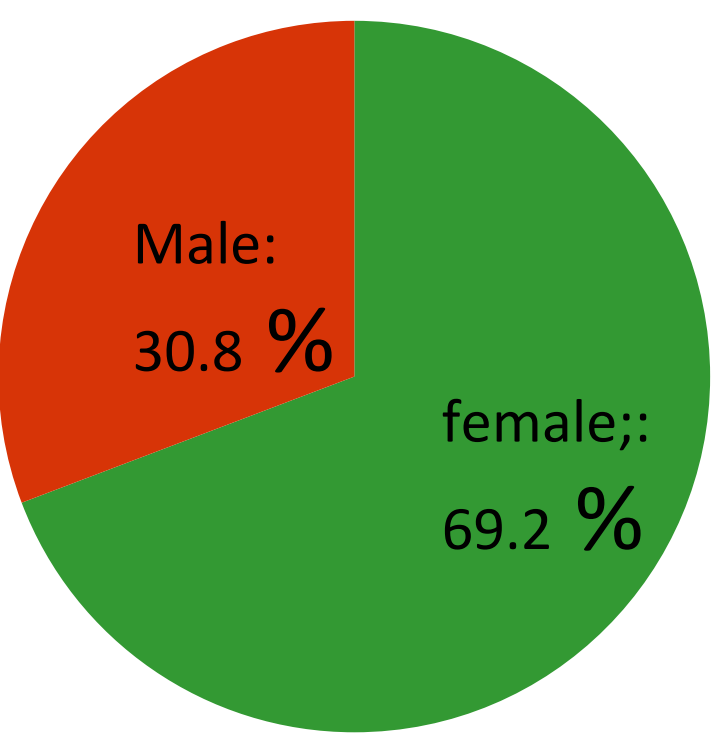

Chart I : Répartition by newborn sex

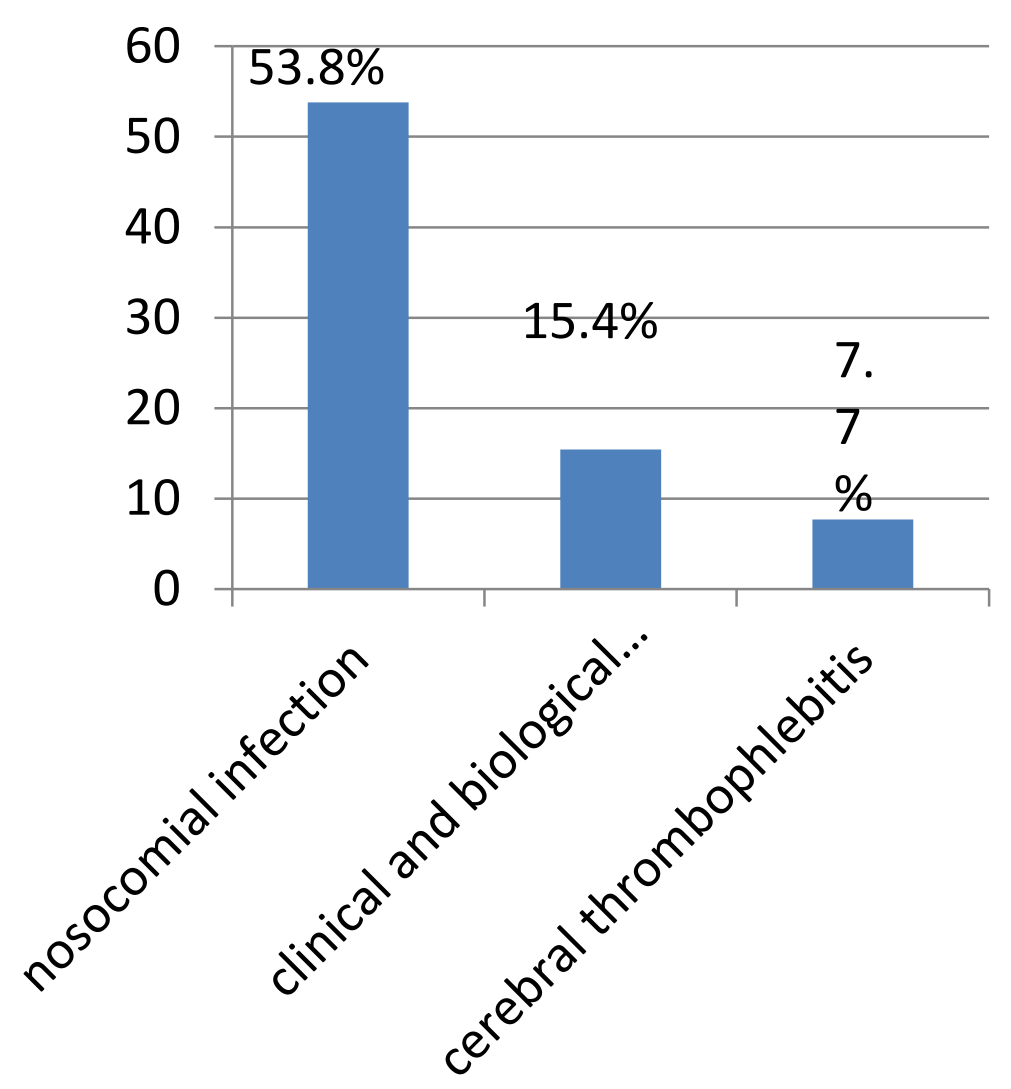

Chart II : Indication of ciprofloxacin prescription

\section{Discussion:}

- Quinolone are a class of synthetic antibiotics with remarkable pharmacological properties and an antibacterial spectrum that has continued to grow with the new generations. They have broad therapeutic indications but their main limitation is their contraindication in children because of the risk of occurrence of cartilaginous lesions in the child in the growth phase.

- In newborns, it is sometimes necessary to break this contraindication when conventional antibiotic therapy has failed, when the germ involved in

-neonatal infection and especially infections of nosocomial origin is multi-resistant to authorized antibiotics and / or when life-threatening is involved.

- Several medical publications promote the use of quinolones in pediatrics and in particular neonatology given the lack of tangible evidence of a specific toxicity of quinolones in children, without declaring their total safety, be ordered to reserve them for treatment second-line in specific situations, and only under close control.

- Ciprofloxacin may be a safe and effective alternative for neonatal infections that are resistant to standard treatments, despite its effects.

-side effects, and whose imputability to ciprofloxacin is uncertain.

-Its use must be carefully weighed and remain exceptional. By drastically limiting their pediatric use, we will help to limit the emergence of resistance.

Conclusion:

Fluoroquinolone must remain in the newborn a second or third-line antibiotic in severe nosocomial infections or no other antibiotic is possible. Their side effects are rare, moderate and reversible.
-In our series, the 13 newborns had no side effects in intra-hospital also short term with a decline of at least 3 months.

- At the end of the treatment, 9 newborns were declared cured against 4 deceased cases. 